\title{
ON CERTAIN VARIETIES WHOSE CURVE SECTIONS ARE HYPERELLIPTIC CURVES*
}

\author{
BY B. C. WONG
}

The properties of hyperelliptic curves, which have been described by Bobek, $\uparrow$ are well known. One important property of such a curve, which must be of genus greater than unity, is that it carries on it one and only one complete and special series of groups of two points. From this property various others follow. For example, a hyperelliptic curve in any space can be transformed into a plane curve of order $n$ with an $(n-2)$-fold point. Now surfaces in a space of any number of dimensions whose sections by spaces of dimension one lower are hyperelliptic curves have certain properties and these have been investigated by Castelnuovo. $\ddagger$ Such a surface must contain $\infty^{1}$ conics such that through each point of it passes one and only one of them. It can be transformed or projected into one, of order $n$, in a 3-dimensional space having an $(n-2)$-fold line; and it is rational.

In this note we call attention to two varieties, in a higher space, whose curve sections are, as we shall show, hyperelliptic curves. One is the $V_{n}^{2 n+1}$ in $S_{2 n+1}$ which was the subject of an investigation by Babbage $\S$ and the other is the $V_{k}{ }^{2 n-2 k+1}$ in $S_{n}$ which is the residual intersection of $(n-k)$ cubic hypersurfaces having in common the intersection $M_{n-2}^{4}$ of two quadric hypersurfaces of $S_{n}$. We shall describe in some detail the surface $V_{k}{ }^{2 n-2 k+1}$ for $k=2$ or $F^{2 n-3}$ and also its projections in a 3-space. Incidentally, we shall obtain a property concerning linear spaces

* Presented to the Society, November 30, 1935.

$\dagger$ Bobek, Ueber hyperelliptische Curven, Mathematischen Annalen, vol. 29 (1887), pp. 386-412.

† Castelnuovo, Sulle superficie algebriche le cui sezioni piane sono curve iperellittiche, Rendiconti del Circolo Matematico di Palermo, vol. 4 (1890), pp. 73-88.

$\S$ Babbage, $A$ series of rational loci with one apparent double point, Proceedings of the Cambridge Philosophical Society, vol. 27 (1931), pp. 399-403. See also B. C. Wong, On a certain rational $V_{n}^{2 n+1}$ in $S_{2 n+1}$, American Journal of Mathematics, vol. 56 (1934), pp. 219-224. 
contained in a general variety whose curve sections are hyperelliptic curves.

We may readily infer that any variety, $V_{t}$, of $t$ dimensions in an $r$-space $S_{r}$ with hyperelliptic curve sections must contain a rational $\infty^{1}$-system of quadric $(t-1)$-dimensional varieties such that through each point of it passes one and only one of them. A general $S_{r-t+1}$ of $S_{r}$ meets each of these quadric varieties in a pair of points and the $\infty^{1}$ pairs of points so obtained form a series of groups of two points on the curve in which $S_{r-t+1}$ meets $V_{t}$. We see also that the variety is rational, in the sense that the coordinates of a point on it are rational functions of $t$ non-homogeneous parameters, $t-1$ of which are the parameters of a point on one of the quadric varieties contained in $V_{t}$ and the other is that of the variable quadric variety of the $\infty^{1}$-system.* Any section $V_{h}$ for $h \leqq 2$ of the variety is also rational in this sense. Again, the variety can be transformed or projected into one, of order $n$, in a $(t+1)$-space $S_{t+1}$ with an $(n-2)$-fold $(t-1)$-space so that any section of the projected variety by a 3 -space of $S_{t+1}$ will be a surface with an $(n-2)$-fold line.

Now we derive a property concerning linear spaces contained in $V_{t}$. It is known $\dagger$ that a quadric variety of $t-1$ dimensions in a $t$-space contains $\infty^{N_{m, t-1}} m$-spaces, where

$$
N_{m, t-1}=\frac{1}{2}(m+1)(2 t-3 m-2),
$$

and

$$
m \leqq \frac{1}{2}(t-2), \text { if } t \text { is even; } \quad m \leqq \frac{1}{2}(t-1), \text { if } t \text { is odd } .
$$

Since $V_{t}$ contains $\infty^{1}$ such quadric varieties, it contains $\infty^{N_{m, t-1+1}} m$-spaces. If $t$ is even and $m=(t-2) / 2$ and therefore $t=2 m+2$, we have $\infty\left(m^{2}+3 m+4\right) / 2 m$-spaces on $V_{2 m+2}$; and, if $t$ is odd and $m=(t-1) / 2$ and therefore $t=2 m+1$, we have

\footnotetext{
* We do not know whether every such $V_{t}$ can be mapped upon a $t$-space.

$\dagger$ Bertini, Projektive Geometrie Mehrdimensionaler Räume, 1924, pp. 140141.
} 
$\infty\left(m^{2}+m+2\right) / 2 m$-spaces on $V_{2 m+1}$. Thus, $V_{2}$ contains $\infty^{2}$ points; $V_{3}$ contains $\infty^{2}$ lines; ${ }^{*} V_{4}$ contains $\infty^{4}$ lines; and so on.

Let us now consider the varieties, $V_{n}^{2 n+1}$ in $S_{2 n+1}$ and $V_{k}^{2 n-2 k+1}$ in $S_{n}$, already mentioned above. The first one, $V_{n}^{2 n+1}$ in $S_{2 n+1}$, as was shown by Babbage, can be represented upon an $S_{n}$ by means of the cubic hypersurfaces of $S_{n}$ passing through the intersection $M_{n-2}^{4}$ of two quadric hypersurfaces of $S_{n}$. It can be shown without difficulty that any $n-k$ of the cubic hypersurfaces intersect in a $V_{k}^{2 n-2 k+1}$ which has a $V_{k-1}^{4(n-k)}$ in common with $M_{n-2}^{4}$. To $V_{k}^{2 n-2 k+1}$ corresponds a section, $V_{k}^{2 n+1}$, of $V_{n}^{2 n+1}$ by an $S_{n+k+1}$ and to $V_{k-1}^{4(n-k)}$ corresponds a ruled variety $V_{k}^{4 k(n-k)}$ on $V_{n}^{2 n+1}$. Now $V_{n}^{2 n+1}$, as was shown by Babbage, has $\infty^{1}$ quadric $(n-1)$-dimensional varieties each of which corresponds to a quadric hypersurface in $S_{n}$ passing through $M_{n-2}^{4}$. Hence, $V_{n}^{2 n+1}$, and therefore any section $V_{k}^{2 n+1}$ of it, has hyperelliptic curve sections. We infer that the $V_{k}^{2 n-2 k+1}$ in $S_{n}$ to which $V_{k}^{2 n+1}$ corresponds must also have such sections. This result also follows from the fact that any quadric hypersurface in $S_{n}$ passing through $M_{n-2}^{4}$ meets $V_{k}^{2 n-2 k+1}$ in a quadric $(k-1)$-dimensional variety besides the $V_{k}^{4(n-k)}$ which is on $M_{n-2}^{4}$. Then, $V_{k}^{2 n-2 k+1}$ has $\infty^{1}$ quadric $(k-1)$-dimensional varieties such that each point on it is on one of them.

The characteristics of $V_{n}{ }^{2 n+1}$ are known, $\dagger$ and those of a surface section, $F^{2 n+1}$, by an $S_{n+3}$ can be easily calculated. The projection of $F^{2 n+1}$ in an $S_{4}$ has $(n-1)^{2}+(n-2)^{2}$ improper double points and the projection in $S_{3}$ has a double curve of order $n^{2}+(n-1)^{2}$ upon which lie $8(n-1)$ pinch points and $(2 n-3)\left(2 n^{2}-6 n+7\right) / 3$ triple points.

The surface, $F^{2 n-3}$, of intersection of $n-2$ cubic hypersurfaces of $S_{n}$ passing through $M_{n-2}^{4}$, to which corresponds the surface of the preceding paragraph, has hyperelliptic sections. According to Castelnuovo, it can be represented upon a plane $f$ by an $\infty^{n}$-system of $n$-ic curves having one $(n-2)$-fold base point, $A$, and $2 n-1$ simple points, $B_{i},(i=1,2, \cdots, 2 n-1)$. From this representation we see that the surface is of class $8 n-12$, that

* We do not know whether any 3-dimensional variety that has rational surface sections contains $\infty^{2}$ lines. A surface with rational curve sections is ruled and, in general, a $V_{t}$ with rational curve sections is the locus of $\infty^{1}(t-1)$ spaces; but a $V_{t}$ with rational surface section is not the locus of $\infty^{2}(t-2)$-spaces.

$\dagger$ B. C. Wong, On a certain rational $V_{n}^{2 n+1}$ in $S_{2 n+1}$, loc. cit. 
its projection in an $S_{4}$ has $2(n-3)(n-4)$ improper double points, and that its projection in an $S_{3}$ has a double curve of order $2(n-2)(n-3)$ with $8 n-24$ pinch points. The image in $f$ of the double curve is a curve of order $(n-3)(2 n-1)$ passing through $A(n-3)(2 n-5)$ times and through the $2 n-1$ points $B_{i}$ each $2(n-3)$ times.

The surface $F^{2 n-3}$ in $S_{n}$ may be regarded as the projection of an $F^{4 n-4}$ in an $S_{3 n-1}$ from an $S_{2 n-2}$ determined by $2 n-1$ general points upon it. The projection of this $F^{4 n-4}$ in $S_{4}$ has $8 n^{2}-31 n+31$ improper double points and the projection in $S_{3}$ has $16 n-28$ pinch points and a double curve of order $8 n^{2}-23 n+17$. $F^{4 n-4}$ is normal in $S_{3 n-1}$ as it is representable upon $f$ by the $\infty^{3 n-1}$-systems of $n$-ic curves having one $(n-2)$ fold base point at $A$ and no other base points.

If we project $F^{2 n-3}$ in $S_{n}$ upon an $S_{3}$ of $S_{n}$ from an $S_{n-4}$ determined by $n-3$ general points on it, we have for projection an $F^{n}$ with an $(n-2)$-fold line which constitutes the double curve of order $(n-2)(n-3) / 2$. On this $(n-2)$-fold line are $4 n-12$ pinch points.

In order to see better how this $(n-2)$-fold line on $F^{2}$ arises from projection, we consider the $F^{n+2}$ in $S_{5}$ which is the projection of $F^{2 n-3}$ from $3 n-6$ points on it. Its representation in $f$ is effected by means of the $\infty^{5} n$-ic curves passing through a given point $A n-2$ times and through each of $3 n-6$ other given points $B_{1}, \cdots, B_{3 n-6}$ once. There are $\infty^{2}$ curves of order $n-1$ in $f$ having $A$ for $(n-3)$-fold point and $B_{1}, \cdots, B_{3 n-6}$ for simple points. Each of these curves goes into a curve, $K^{n}$, of order $n$ on $F^{n+2}$, which, constituting with every conic of the surface a 4-space section of the surface, is a 3-space curve. Hence, there are $\infty^{2}$ such 3 -space curves on $F^{n+2}$. Each of these curves lies on a quadric surface and meets the generators of one regulus $n-2$ times and the generators of the other regulus twice, and therefore it has $\infty^{1}(n-2)$-secant lines. Through a general point $P$ pass $\infty^{1}$ such curves all having an $(n-2)$-secant line in common and the 3 -spaces containing them all pass through this common $(n-2)$-secant line. These 3 -spaces through $P$ form a quadric hypersurface $V_{4}^{2}$ of $S_{5}$.

Now project this $F^{n+2}$ from $P$ upon an $S_{4}$ and the projection, $F^{n+1}$, has an improper multiple point of order $n-3$ which is to be regarded as the union of $(n-3)(n-4) / 2$ improper double points 
and which is the intersection of the common $(n-2)$-secant line of the $\infty^{1} 3$-space $n$-ic curves on $F^{n+2}$ through $P$. On $F^{n+1}$ are $\infty^{1}$ plane curves of order $n-1$ all having an $(n-3)$-fold point at the improper $(n-3)$-fold point. These $\infty^{1}$ curves are the projections of the 3 -space $n$-ic curves through $P$. Now in the plane $f$ of representation there are $3 n-5$ simple base points $B_{1}, \cdots, B_{3 n-5}$ besides the $(n-2)$-fold base point $A$. The pencil of $(n-1)$-ic curves through $A \quad n-3$ times and $B_{i}$ once yields the $\infty^{1}$-system of plane $(n-1)$-ic curves on $F^{n+1}$ with the same $(n-3)$-fold point at the improper $(n-3)$-fold point of the surface. The planes of these curves generate a $V_{3}^{2}$ which is the intersection of the $V_{4}^{2}$, mentioned in the preceding paragraph, and $S_{4}$. Note that the $n-3$ base points of the pencil of $(n-1)$ ic curves of $f$, distinct from $A$ and $B_{i}$, are the images of the improper $(n-3)$-fold point of $F^{n+1}$.

A general section of $F^{n+1}$ by an $S_{3}$ is a curve having $\infty^{1}$ $(n-1)$-secant lines lying on a quadric surface which is the section of $V_{3}^{2}$ by $S_{3}$. This curve meets the generators of one regulus of this surface in $n-1$ points and those of the other in two points. It is the partial intersection of the quadric surface and another surface, of order $2 n-2$, having $n-3$ lines in common.

Now of the $\infty^{1}(n-1)$-ic curves on $F^{n+1}$ one, say $K^{n-1}$, passes through a general point $Q$. Projecting $F^{n+1}$ from $Q$ upon $S_{3}$, we obtain an $F^{n}$ with an $(n-2)$-fold line $l$ which is the projection of $K^{n-1}$. The curve $K^{n-1}$ having an $(n-3)$-fold point is of genus 3 and class $4 n-10$. The point $Q$ being on $K^{n-1}$, the number of tangent lines from $Q$ to $K^{n-1}$ is therefore $4 n-12$. The projections of the points of contact are pinch points on $F^{n}$. Hence $F^{n}$ has $4 n-12$ pinch points on the $(n-2)$-fold line $l$.

If we project $F^{n+1}$ from its improper $(n-3)$-fold point upon $S_{3}$, we obtain for projection a quartic surface composed of two coincident quadric surfaces. This double quadric surface is the intersection of the $V_{3}^{2}$ already mentioned and $S_{3}$.

It is of interest to note that the $F^{4 n-4}$ in $S_{3 n-1}$ may be represented upon $f$ by an $\infty^{3 n-1}$-system of $(n+N)$-ic curves having one $(n+N-2)$-fold base point and $N$ double base points for all values of $N \geqq 0$. A general projection of $F^{4 n-4}$ upon an $S_{4}$ has $8 n^{2}-31 n+31$ improper double points all lying in a plane $\pi$. This plane $\pi$ contains a curve $K^{4 n-6}$, of order $4 n-6$, of the projected surface and this is of genus $n-3$ and has the $8 n^{2}-31 n+31$ im- 
proper double points for nodes. If we project the projected surface upon an $S_{3}$, we have an $F^{\prime 4 n-4}$ having a double curve of order $8 n^{2}-23 n+17$ with $16 n-28$ pinch points. If the center of projection is in $\pi$, the double curve degenerates into a $(4 n-6)$ fold line and $3 n-4$ double lines. Since the class of $K^{4 n-6}$ is $10 n-20$, there are on the $(4 n-6)$-fold line $10 n-20$ pinch points. The remaining $6 n-8$ pinch points are on the $3 n-4$ double lines, 2 on each.

The University of California

\section{SPINORS AND TENSORS}

BY G. Y. RAINICH

It is well known that there are two kinds of quantities connected with the representations of a group of rotations-the tensors and the spinors. ${ }^{*}$ Since the advent of the relativity theory we had been led to believe, in the words of O. Veblen, $\dagger$ "that any physical phenomena could be described by means of tensors." But then came the Dirac equations of the electron which give an example of a situation described in terms of spinors. Does it mean that we have to change the belief expressed above? It does not follow. All that has happened is that we have a phenomenon not described in terms of tensors; that does not mean that it cannot be-so described. That it might be possible to describe every situation given in spinors also in tensors is suggested by the fact that there exist algebraic relations between spinors and tensors; it may be possible to eliminate the spinors from a sufficient number of these algebraic relations and the given spinor differential equations, and obtain in this way an equivalent description in tensors. The discussion of the general case should not be very difficult, but it seemed that a simple special case should be worked out first, and that is why I suggested to Gordon Fuller the problem which he discusses in his article. $\ddagger$ The problem there is treated without

* Compare, for example, R. Brauer and H. Weyl, American Journal of Mathematics, vol. 40 (1935), p. 425.

$\dagger$ Proceedings of the National Academy of Sciences, vol. 24 (1934), p. 282.

$\ddagger$ This issue of this Bulletin, vol. 42 (1936), p. 107. 